\title{
Theoretical Study on Planar Anionic Polysilicon Chains and Cyclic Si6 Anions with D6h Symmetry
}

Masae Takahashi and Yoshiyuki Kawazoe

\section{Contents}

Table S1. Cartesian Coordinates and Absolute Energies of All Optimized Structures 
Table S1. Cartesian Coordinates and Absolute Energies of All Optimized Structures

\begin{tabular}{|c|c|c|c|}
\hline Compound & Method & Energy/a.u. & Cartesian/Å \\
\hline \multirow{16}{*}{$\begin{array}{c}\text { 11a } \\
\text { (singlet) }\end{array}$} & \multirow[t]{4}{*}{ B3LYP/6-311++G(3df,3pd) } & \multirow[t]{4}{*}{-580.1246223} & Si,-0.0009941232,0.,-1.0501316234 \\
\hline & & & Si,0.0009557042,0.,1.050186133 \\
\hline & & & H,1.2255128129,0.,1.9001974585 \\
\hline & & & H,-1.2249749468,0.,-1.900960593 \\
\hline & \multirow[t]{4}{*}{ MP2/6-311++G(3df,3pd) } & \multirow[t]{4}{*}{-579.1000071} & Si,-0.0108404556,0.,-1.0501550004 \\
\hline & & & Si,0.0108369385,0.,1.0501599581 \\
\hline & & & H,1.2221091744,0.,1.9041633542 \\
\hline & & & $\mathrm{H},-1.2220599351,0 .,-1.904232763$ \\
\hline & \multirow[t]{4}{*}{ G2 } & \multirow[t]{4}{*}{-579.219654} & Si,0,0.0121505819,0.,-1.0512530535 \\
\hline & & & $\mathrm{Si}, 0,-0.0121274232,0 ., 1.0512211591$ \\
\hline & & & $\mathrm{H}, 0,1.2056388645,0 ., 1.9118505869$ \\
\hline & & & $\mathrm{H}, 0,-1.2059630856,0 .,-1.911404064$ \\
\hline & \multirow[t]{4}{*}{ CBS-Q } & \multirow[t]{4}{*}{-579.220682} & Si,0,0.0108061584,0.,-1.0530271152 \\
\hline & & & $\mathrm{Si}, 0,-0.0107814426,0 ., 1.0529931191$ \\
\hline & & & $\mathrm{H}, 0,1.2064855426,0 ., 1.9143933273$ \\
\hline & & & H,0,-1.2068315635,0.,-1.9139173828 \\
\hline \multirow{4}{*}{$\begin{array}{c}\text { 11a } \\
\text { (triplet) }\end{array}$} & \multirow[t]{4}{*}{ B3LYP/6-311++G(3df,3pd) } & \multirow[t]{4}{*}{-580.1224552} & Si,0.0013302403,0.,-1.1456573198 \\
\hline & & & Si,-0.0013336922,0.,1.1456698824 \\
\hline & & & H,1.4599402419,0.,1.5294012215 \\
\hline & & & H,-1.4598919156,0.,-1.529577097 \\
\hline \multirow{8}{*}{$\begin{array}{c}11 b \\
\text { (singlet) }\end{array}$} & \multirow[t]{4}{*}{ B3LYP/6-311++G(3df,3pd) } & \multirow[t]{4}{*}{-580.1382098} & Si,0,0.,0.,-0.9720185658 \\
\hline & & & Si,0,0.,0.,1.2291426827 \\
\hline & & & $\mathrm{H}, 0,1.2308374332,0 .,-1.7998688181$ \\
\hline & & & H,0,-1.2308374332,0.,-1.7998688181 \\
\hline & \multirow[t]{4}{*}{ MP2/6-311++G(3df,3pd) } & \multirow[t]{4}{*}{-579.1447549} & Si,0,0.,0.,-0.9740764763 \\
\hline & & & Si, $0,0 ., 0 ., 1.2294472912$ \\
\hline & & & $\mathrm{H}, 0,1.2312000513,0 .,-1.7875957043$ \\
\hline & & & $\mathrm{H}, 0,-1.2312000513,0 .,-1.7875957043$ \\
\hline \multirow{8}{*}{$\begin{array}{c}\text { 11c } \\
\text { (singlet) }\end{array}$} & \multirow[t]{4}{*}{ B3LYP/6-311++G(3df,3pd) } & \multirow[t]{4}{*}{-580.1403684} & Si,0,-0.0709678225,0.,-1.1394821397 \\
\hline & & & $\mathrm{Si}, 0,-0.0508704904,0 ., 0.9723558312$ \\
\hline & & & $\mathrm{H}, 0,0.4662168416,0 ., 2.3688547877$ \\
\hline & & & $\mathrm{H}, 0,1.2395195391,0 .,-0.029086468$ \\
\hline & \multirow[t]{4}{*}{ MP2/6-311++G(3df,3pd) } & -579.1542166 & Si,0,-0.0606812819,0.,-1.144718021 \\
\hline & & & $\mathrm{Si}, 0,-0.0597462719,0 ., 0.9781871476$ \\
\hline & & & H,0,0.4693069051,0.,2.3625466865 \\
\hline & & & H,0,1.2166788478,0.,-0.0311144591 \\
\hline 11d & B3LYP/6-311++G(3df,3pd) & -580.1571318 & Si,0,-0.0314251542,0.0407105283,-1.106396364 \\
\hline (singlet) & & & Si,0,-0.0314970817,0.0406549047,1.106396364 \\
\hline & & & $\mathrm{H}, 0,1.2234280476,0.0359371042,0.0000425123$ \\
\hline & & & $\mathrm{H}, 0,-0.3425167447,-1.1750531659,-0.0000425123$ \\
\hline & MP2/6-311++G(3df,3pd) & -579.1699596 & Si,0,-0.0305878052,0.0396204565,-1.1070882409 \\
\hline & & & $\mathrm{Si}, 0,-0.0306526803,0.0395702867,1.1070882409$ \\
\hline & & & $\mathrm{H}, 0,1.2018674153,0.0435902963,0.0000469822$ \\
\hline & & & $\mathrm{H}, 0,-0.3445006172,-1.1522607008,-0.0000469822$ \\
\hline $12 a$ & B3LYP/6-311++G(3df,3pd) & -580.0893953 & Si,-0.0012784483,0.,-1.1442111764 \\
\hline (singlet) & & & Si, $0.0012784483,0 ., 1.1442111764$ \\
\hline (singlet) & & & H,1.5418223542,0.,1.3618289462 \\
\hline & & & H,-1.5418223542,0.,-1.3618289462 \\
\hline & MP2/6-311++G(3df,3pd) & -579.0941238 & Si,0.0072523208,0.,-1.1393139851 \\
\hline & & & Si,-0.0072523208,0.,1.1393139851 \\
\hline & & & H,1.5226080275,0.,1.3497526416 \\
\hline & & & H,-1.5226080275,0.,-1.3497526416 \\
\hline
\end{tabular}




\begin{tabular}{ccc} 
& CBS-Q & -579.175679 \\
& \\
\hline $\begin{array}{c}\text { 12a } \\
\text { (triplet) }\end{array}$ & B3LYP/6-311++G(3df,3pd) & -580.0551098
\end{tabular}

Si,0,0.0038191497,0.,-1.1458955775 Si, $0,-0.0038191497,0 ., 1.1458955775$ H,0,1.5534013176,0.,1.3568584486 H, $0,-1.5534013176,0,-1.3568584486$

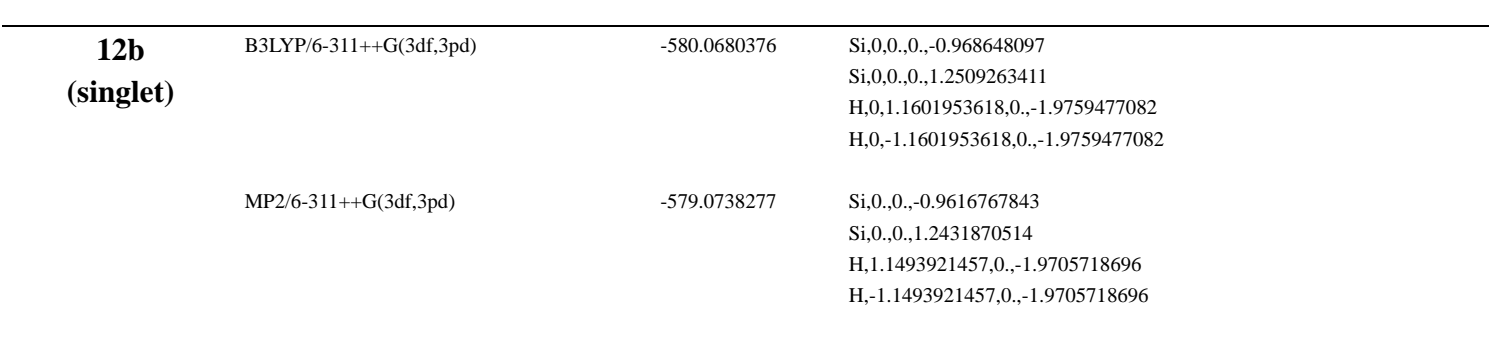

\begin{tabular}{|c|c|c|c|}
\hline $\begin{array}{c}\text { 12c' } \\
\text { (singlet) }\end{array}$ & B3LYP/6-311++G(3df,3pd) & -580.084339 & $\begin{array}{l}\text { Si,-0.1013456541,0.,-1.1536539977 } \\
\text { Si,-0.1013456541,0.,1.1536539977 } \\
\text { H,1.4188391567,0.,15106809476 } \\
\text { H,1.4188391567,0.,-1.5106809476 }\end{array}$ \\
\hline & MP2/6-311++G(3df,3pd) & -579.0880314 & $\begin{array}{l}\text { Si,-0.1009934324,0.-1.1488173652 } \\
\text { Si,-0.1009934324,0.,1.1488173652 } \\
\text { H,1.4139080541,0.,1.4704512623 } \\
\text { H,1.4139080541,0.,-1.4704512623 }\end{array}$ \\
\hline $\begin{array}{c}\text { 12d } \\
\text { (singlet) }\end{array}$ & B3LYP/6-311++G(3df,3pd) & -580.0138077 & $\begin{array}{l}\text { Si,0,0.0118854578,0.0464011062,-1.2504388483 } \\
\text { Si,0,-0.0477600571,-0.0036474235,1.2504388483 } \\
\text { H,0,1.0458223428,0.3675568217,0.0322038637 } \\
\text { H,0,-0.5435779519,-0.966108379,-0.0322038637 }\end{array}$ \\
\hline & MP2/6-311++G(3df,3pd) & -579.0040629 & $\begin{array}{l}\text { Si,-0.005082196,0.063958585,-1.19304213 } \\
\text { Si,-0.0621043963,0.0161112777,1.19304213 } \\
\text { H,1.1871854768,0.0410441431,0.0289659895 } \\
\text { H,-0.246573185,-1.1620222211,-0.0289659895 }\end{array}$ \\
\hline $\mathrm{Si}_{4} \mathbf{H}_{2}$ & B3LYP/6-311++G(d,p) & -1159.0806339 & $\begin{array}{l}\text { Si,-1.052393411,0.,-2.6415729695 } \\
\text { Si,-1.025536558,0.,-0.5247477273 } \\
\text { Si,1.0255544064,0.,0.524747319 } \\
\text { Si,1.0523814604,0.,2.6415681721 } \\
\text { H,2.1730617875,0.,3.6266681403 } \\
\text { H,-2.1731443562,0.,-3.6265952603 }\end{array}$ \\
\hline & MP2/6-311++G(d,p) & -1157.0483232 & $\begin{array}{l}\text { Si,-1.0352427954,0.,-2.6455218105 } \\
\text { Si,-1.0090653912,0.,-0.5331845345 } \\
\text { Si,1.0091839014,0.,0.5331735732 } \\
\text { Si,1.0351361261,0.,2.6455271514 } \\
\text { H,2.1723485329,0.,3.5947740159 } \\
\text { H,-2.1725143056,0.,-3.5946953316 }\end{array}$ \\
\hline $\mathrm{Si}_{4} \mathrm{H}_{2}{ }^{2-}$ & B3LYP/6-311++G(d,p) & -1159.1392981 & $\begin{array}{l}\text { Si,-0.6526719891,-0.0002898195,-3.1028401012 } \\
\text { Si,-0.7336128589,-0.0001759078,-0.8542966683 } \\
\text { Si,0.733598661,0.0001210598,0.8542973854 } \\
\text { Si,0.6526851484,0.0003749861,3.1028408801 } \\
\text { H,2.1993241744,0.0002889774,3.2104744624 } \\
\text { H,-2.1993096332,-0.0007134383,-3.2104954045 }\end{array}$ \\
\hline & MP2/6-311++G(d,p) & -1157.0732734 & $\begin{array}{l}\text { Si,-0.6487452325,-0.0003232936,-3.0739573683 } \\
\text { Si,-0.7384505405,-0.0001469161,-0.8312447157 } \\
\text { Si,0.738415659,0.0001298088,0.8312464551 } \\
\text { Si,0.6487775468,0.0003338149,3.0739593408 } \\
\text { H,2.1783071432,0.0005467928,3.1923452666 } \\
\text { H,-2.1782712026,-0.0004545882,-3.1923972341 }\end{array}$ \\
\hline
\end{tabular}


B3LYP/6-311++G(3df,3pd)

MP2/6-311++G(3df,3pd)
$-1159.1507215$

$-1157.1949466$

Si,-0.6526056732,-0.0002813365,-3.1049979665 Si,-0.7107867503,-0.0001844433,-0.8649787791 Si, $0.7107829784,0.0001099934,0.8649789719$ Si, $0.652609171,0.0003838708,3.1049981866$ H,2.1953911059,0.0003025287,3.1915656795 H,-2.1953872694,-0.0006957108,-3.1915714615

Si,-0.6444962767,-0.0003201865,-3.1122541174 Si,-0.6897520342,-0.0001603242,-0.8665104366 Si,0.689741628,0.0001275741,0.8665110963 $\mathrm{Si}, 0.6445059712,0.0003402472,3.1122546458$ $\mathrm{H}, 2.1793443248,0.0005842126,3.1459671314$ H,-2.179334360d1,-0.0004065617,-3.1459837651

Si,-1.002910444,0.0000468786,-2.9855941046 Si,-1.0426227719,0.000086064,0.6325573299 Si, $1.0426221537,-0.0000817848,0.6325573061$ Si,1.0029110182,-0.0000611433,2.9855941878 H,2.5420105525,0.0001162607,3.1534160308 H,-2.5420099367,0.0000235357,-3.1534168617

MP2/6-311++G(d,p)

MP2/6-311++G(3df,3pd)
$-1156.5464417$

Si,-1.0754541054,-0.0000229721,-2.9035385815 Si,-1.0087760299,0.0000103561,-0.6000670309 Si,1.008760137,-0.0000073426,0.6000687037 Si,1.0754688897,0.0000200595,2.9035383197 H,2.5911084812,-0.0000154676,3.0257193818 H,-2.5910929619,0.0000140562,-3.0257391361

$-1158.6352647$

Si,-0.9957095906,0.000052172,-2.966769238 Si,-1.0263279665,-0.0000345672,-0.6330710127 Si,1.0263285885,-0.0000026177,0.633070892 Si,0.9957090239,-0.0000548249,2.9667692539 H,2.5271688683,0.0003046342,3.129713292 H,-2.5271696433,0.0002530926,-3.1297118262

$-1156.6801482$ Si,-0.9776659226,-0.0000249638,-2.9411708996 Si,-0.9908098834,0.0002185708,-0.6296275655 Si, $0.9908036183,-0.0000590831,0.6296279516$ Si,0.9776717474,-0.0001277399,2.9411710859 H,2.4963554288,0.000012238,3.0728935311 H,-2.4963492655,-0.0001072143,-3.0729015455

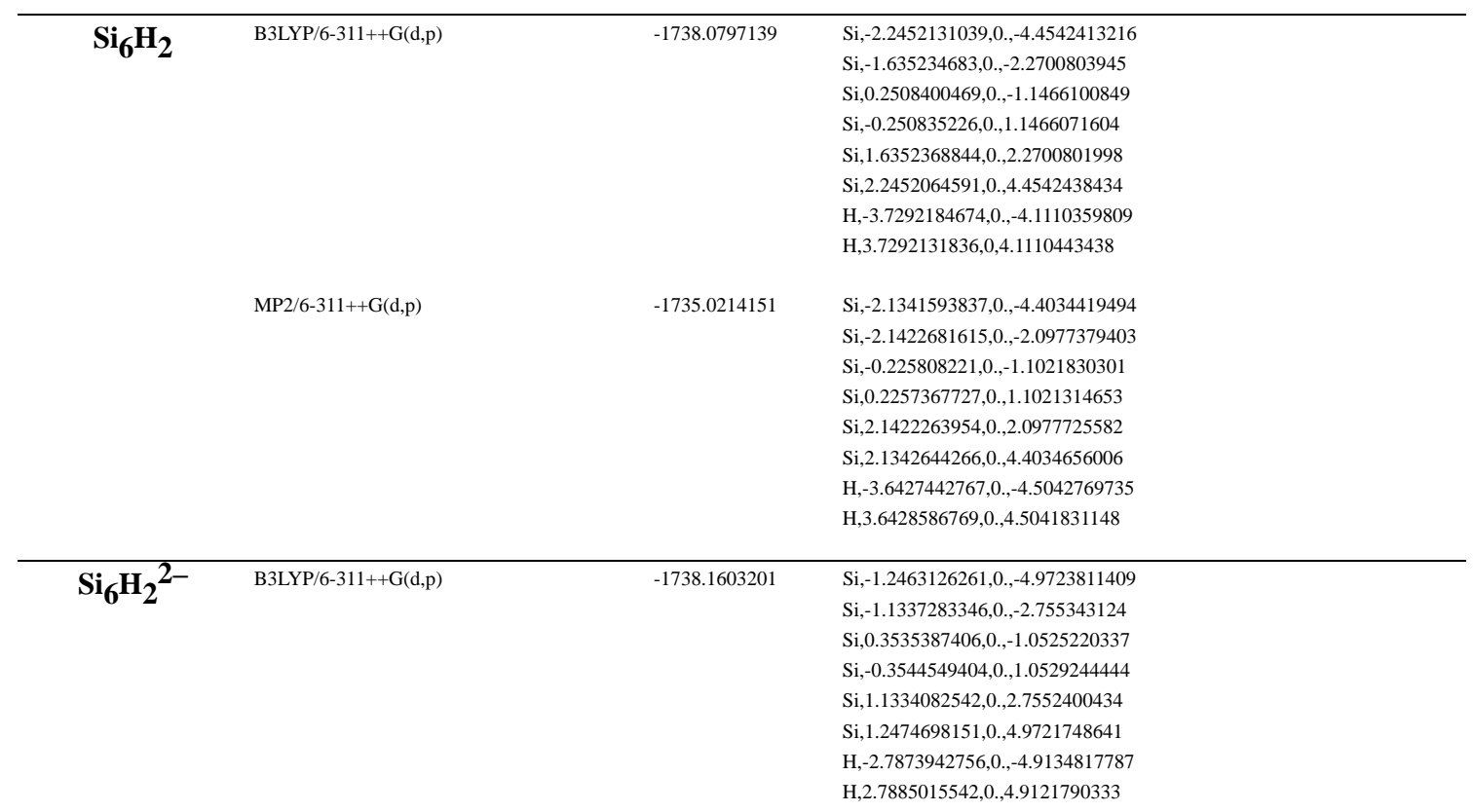




\begin{tabular}{|c|c|c|c|}
\hline \multirow{24}{*}{$\mathrm{Si}_{6} \mathrm{H}_{2}{ }^{4-}$} & \multirow[t]{8}{*}{ B3LYP/6-311++G(d,p) } & \multirow[t]{8}{*}{-1737.7530131} & $\mathrm{Si},-2.8044631595,0 .,-4.2324266257$ \\
\hline & & & Si,-2.7614748389,0.,-1.9005329648 \\
\hline & & & Si,-0.5095855924,0.,-0.966790641 \\
\hline & & & $\mathrm{Si}, 0.5095847636,0 ., 0.9667916714$ \\
\hline & & & $\mathrm{Si}, 2.7614747816,0 ., 1.9005325057$ \\
\hline & & & $\mathrm{Si}, 2.804463986,0 ., 4.2324261225$ \\
\hline & & & Н,-4.3625746999,0.,-4.2270456264 \\
\hline & & & H,4.3625755358,0.,4.2270446751 \\
\hline & \multirow[t]{8}{*}{ MP2/6-311++G(d,p) } & \multirow[t]{8}{*}{-1734.6701516} & Si,-2.7534828547,0.,-4.1359350591 \\
\hline & & & Si,-2.7486573142,0.,-1.8309440552 \\
\hline & & & Si,-0.5239090719,0.,-0.961305686 \\
\hline & & & Si,0.5239080016,0.,0.9613035083 \\
\hline & & & $\mathrm{Si}, 2.7486607382,0 ., 1.8309452834$ \\
\hline & & & Si,2.7534806431,0.,4.1359360257 \\
\hline & & & H,-4.2892516451,0.,-4.1752376764 \\
\hline & & & H,4.2892496566,0.,4.1752374385 \\
\hline & \multirow[t]{8}{*}{ B3LYP/6-311++G(3df,3pd) } & \multirow[t]{8}{*}{-1737.7697419} & Si,-2.713293377,0.,-4.2601733949 \\
\hline & & & Si,-2.7139187531,0.,-1.9427827111 \\
\hline & & & $\mathrm{Si},-0.5090515766,0 .,-0.9608892203$ \\
\hline & & & $\mathrm{Si}, 0.509051521,0 ., 0.9608892548$ \\
\hline & & & Si,2.7139187264,0,1.9427826979 \\
\hline & & & $\mathrm{Si}, 2.7132934535,0 ., 4.2601733816$ \\
\hline & & & Н,-4.2624324613,0.,-4.2927372629 \\
\hline & & & H,4.2624325406,0.,4.2927371496 \\
\hline \multirow{16}{*}{$\mathrm{Si}_{6} \mathrm{H}_{2}{ }^{6-}$} & \multirow[t]{8}{*}{ B3LYP/6-311++G(d,p) } & \multirow[t]{8}{*}{-1736.9754455} & Si,1.8521965107,-5.1235394217,0. \\
\hline & & & Si,1.8508199413,-2.7160838632,0. \\
\hline & & & Si,-0.0563284898,-1.1705606117,0. \\
\hline & & & Si,0.0563279072,1.1705603427,0. \\
\hline & & & Si,-1.8508202315,2.7160839665,0. \\
\hline & & & Si,-1.852195699,5.1235395069,0. \\
\hline & & & Н,3.4043708721,-5.198020432,0. \\
\hline & & & H,-3.4043700171,5.1980215594,0. \\
\hline & \multirow[t]{8}{*}{ B3LYP/6-311++G(3df,3pd) } & \multirow[t]{8}{*}{-1737.0005652} & Si,1.8340082504,-5.0799537478,0. \\
\hline & & & Si,1.7517086451,-2.7053262117,0. \\
\hline & & & $\mathrm{Si},-0.0984464416,-1.1566823411,0$. \\
\hline & & & Si,0.098447321,1.1566827495,0. \\
\hline & & & Si,-1.7517081655,2.7053260712,0. \\
\hline & & & Si,-1.834009522,5.0799535689,0. \\
\hline & & & Н,3.3675635568,-5.0497994805,0. \\
\hline & & & Н,-3.367564782,5.0497982346,0. \\
\hline \multirow{10}{*}{$\mathrm{Si}_{8} \mathrm{H}_{2}$} & \multirow[t]{10}{*}{ MP2/6-311++G(d,p) } & \multirow[t]{10}{*}{-2312.9886417} & $\mathrm{Si},-3.4638092759,0 .,-5.7728336962$ \\
\hline & & & Si,-3.4690693035,0.,-3.4845285615 \\
\hline & & & Si,-1.5369769209,0.,-2.5305726038 \\
\hline & & & Si,-1.0251494552,0.,-0.3458370927 \\
\hline & & & Si,1.0251494552,0.,0.3458370927 \\
\hline & & & Si,1.5369769209,0.,2.5305726038 \\
\hline & & & $\mathrm{Si}, 3.4690693035,0 ., 3.4845285615$ \\
\hline & & & Si,3.4638092759,0.,5.7728336962 \\
\hline & & & H,-4.9694009997,0.,-5.9058653063 \\
\hline & & & H,4.9694009997,0.,5.9058653063 \\
\hline
\end{tabular}




\begin{tabular}{|c|c|c|c|}
\hline \multirow{20}{*}{$\mathrm{Si}_{8} \mathrm{H}_{2}{ }^{2-}$} & \multirow[t]{10}{*}{ B3LYP/6-311++G(d,p) } & \multirow[t]{10}{*}{-2317.1668926} & Si,-0.0863458409,0.,-6.9160487423 \\
\hline & & & Si,-0.0521085954,0.,-4.7167149564 \\
\hline & & & Si,1.1477777438,0.,-2.8022195387 \\
\hline & & & Si,-0.2673630997,0.,-1.0976714855 \\
\hline & & & Si,0.2673630997,0.,1.0976714855 \\
\hline & & & Si,-1.14777774438,0.,2.8022195387 \\
\hline & & & Si,0.0521085954,0.,4.7167149564 \\
\hline & & & $\mathrm{Si}, 0.0863458409,0 ., 6.9160487423$ \\
\hline & & & H,-1.6221229135,0.,-6.9298476908 \\
\hline & & & H,1.6221229135,0.,6.9298476908 \\
\hline & \multirow[t]{10}{*}{ MP2/6-311++G(d,p) } & \multirow[t]{10}{*}{-2313.1104007} & Si,-2.6744467996,0.,-4.5462863752 \\
\hline & & & Si,-1.0274721315,0.,-3.108532535 \\
\hline & & & Si,1.3096316218,0.,-3.0305845341 \\
\hline & & & Si,0.2396540999,0.,-1.083380142 \\
\hline & & & Si,-0.2396540999,0.,1.083380142 \\
\hline & & & Si,-1.3096316218,0.,3.0305845341 \\
\hline & & & Si,1.0274721315,0.,3.108532535 \\
\hline & & & Si,2.6744467996,0.,4.5462863752 \\
\hline & & & H,-3.8905152657,0.,-3.6435843669 \\
\hline & & & Н,3.8905152657,0.,3.6435843669 \\
\hline \multirow{30}{*}{$\mathrm{Si}_{\mathbf{8}} \mathrm{H}_{2}{ }^{4-}$} & \multirow[t]{10}{*}{ B3LYP/6-311++G(d,p) } & \multirow[t]{10}{*}{-2316.8423507} & Si,-3.8939980057,0.,-5.8441565999 \\
\hline & & & Si,-3.7859451938,0.,-3.5408155562 \\
\hline & & & Si,-1.8139126931,0.,-2.3223179291 \\
\hline & & & Si,-1.1975024538,0.,-0.1696187448 \\
\hline & & & Si,1.1975024538,0.,0.1696187448 \\
\hline & & & Si,1.8139126931,0.,2.3223179291 \\
\hline & & & Si,3.7859451938,0.,3.5408155562 \\
\hline & & & Si,3.8939980057,0.,5.8441565999 \\
\hline & & & H,-5.4528920536,0.,-5.8322989961 \\
\hline & & & H,5.4528920536,0.,5.8322989961 \\
\hline & \multirow[t]{10}{*}{ MP2/6-311++G(d,p) } & \multirow[t]{10}{*}{-2312.7512188} & Si,-3.8902289663,0.,-5.7581154546 \\
\hline & & & Si,-3.8978695755,0.,-3.4603064094 \\
\hline & & & Si,-1.7397918149,0.,-2.5710718894 \\
\hline & & & Si,-1.0365155761,0.,-0.4785031508 \\
\hline & & & Si,1.0365155761,0.,0.4785031508 \\
\hline & & & Si,1.7397918149,0.,2.5710718894 \\
\hline & & & Si,3.8978695755,0.,3.4603064094 \\
\hline & & & Si,3.8902289663,0.,5.7581154546 \\
\hline & & & H,-5.4292350282,0.,-5.8013038279 \\
\hline & & & H,5.4292350282,0.,5.8013038279 \\
\hline & \multirow[t]{10}{*}{ B3LYP/6-311++G(3df,3pd) } & \multirow[t]{10}{*}{-2316.863934} & Si,-3.6010774879,0.,-6.0031202194 \\
\hline & & & Si,-3.555592358,0.,-3.7113031986 \\
\hline & & & Si,-1.7036366943,0.,-2.3521519932 \\
\hline & & & Si,-1.1949666879,0.,-0.1742755682 \\
\hline & & & Si,1.1949666879,0.,0.1742755682 \\
\hline & & & Si,1.7036366943,0.,2.3521519932 \\
\hline & & & Si,3.555592358,0.,3.7113031986 \\
\hline & & & Si,3.6010774879,0.,6.0031202194 \\
\hline & & & H,-5.1525324983,0.,-6.03998785 \\
\hline & & & H,5.1525324983,0.,6.03998785 \\
\hline \multirow{10}{*}{$\mathrm{Si}_{8} \mathrm{H}_{2}{ }^{6-}$} & \multirow[t]{10}{*}{ B3LYP/6-311++G(d,p) } & \multirow[t]{10}{*}{-2316.1529409} & Si,-2.6500089008,0.,-6.8668179908 \\
\hline & & & Si,-2.6877034745,0.,-4.4911064775 \\
\hline & & & Si,-0.5645802934,0.,-3.2168590326 \\
\hline & & & Si,-0.7535495479,0.,-0.9154372892 \\
\hline & & & Si,0.7535495479,0.,0.9154372892 \\
\hline & & & Si,0.5645802934,0.,3.2168590326 \\
\hline & & & Si,2.6877034745,0.,4.4911064775 \\
\hline & & & Si,2.6500089008,0.,6.8668179908 \\
\hline & & & H,-4.19138062,0.,-6.9614890002 \\
\hline & & & H,4.19138062,0.,6.9614890002 \\
\hline
\end{tabular}


MP2/6-311++G(d,p)

B3LYP/6-311++G(3df,3pd)
$-2312.0166839$

$-2316.1792127$
Si,-0.6302047947,0.,--7.5136044975 Si,-0.8745960267,0.,-5.0904368151 Si,-0.1095885427,0.,-3.0065865626 Si,-0.9341869579,0.,-0.7188737628 Si, $0.9341869579,0 ., 0.7188737628$ Si, $0.1095885427,0 ., 3.0065865626$ $\mathrm{Si}, 0.8745960267,0 ., 5.0904368151$ Si,0.6302047947,0.,7.5136044975 H,-2.1503860831,0.,-7.7440118754 H,2.1503860831,0.,7.7440118754

Si,-2.7429916852,0.,-6.8043085916 Si,-2.7331840677,0.,-4.451247245 Si,-0.6601918371,0.,-3.1664936332 Si,-0.7783369125,0.,-0.8840861958 Si, $0.7783369125,0,0.8840861958$ $\mathrm{Si}, 0.6601918371,0 ., 3.1664936332$ Si,2.7331840677,0.,4.451247245 Si,2.7429916852,0.,6.8043085916 H,-4.2742135866,0.,-6.8548926525 H,4.2742135866,0.,6.8548926525

\begin{tabular}{|c|c|c|c|}
\hline$c-\mathrm{Si}_{6}{ }^{2-}$ & B3LYP/6-311++G(d,p) & -1736.9257021 & $\begin{array}{l}\text { Si,-1.9377420074,0.,-1.1187558696 } \\
\text { Si,-1.9377420074,0.,1.1187558696 } \\
\text { Si,0.,0.,2.2375117391 } \\
\text { Si,1.9377420074,0.,1.1187558696 } \\
\text { Si,1.9377420074,0.,-1.1187558696 } \\
\text { Si,0.,0.,-2.2375117391 }\end{array}$ \\
\hline & MP2/6-311++G(d,p) & -1733.9795251 & $\begin{array}{l}\text { Si,-1.938712485,0.,-1.1193161751 } \\
\text { Si,-1.938712485,0.,1.1193161751 } \\
\text { Si,0.,0.,2.2386323501 } \\
\text { Si,1.938712485,0.,1.1193161751 } \\
\text { Si,1.938712485,0.,-1.1193161751 } \\
\text { Si,0.,0.,-2.2386323501 }\end{array}$ \\
\hline & MP2/6-311++G(3df,3pd) & -1734.1583078 & $\begin{array}{l}\text { Si,-1.9406363477,0.,-1.1204269177 } \\
\text { Si,-1.9406363477,0.,1.1204269177 } \\
\text { Si,0.,0.,2.2408538355 } \\
\text { Si,1.9406363477,0.,1.1204269177 } \\
\text { Si,1.9406363477,0.,-1.1204269177 } \\
\text { Si,0.,0.,-2.2408538355 }\end{array}$ \\
\hline$c-\mathrm{Si}_{6}{ }^{4-}$ & B3LYP/6-311++G(d,p) & -1736.4867094 & $\begin{array}{l}\text { Si,-1.8061359193,0.0005220339,-1.1295854271 } \\
\text { Si,-1.8061416801,0.0004445466,1.1297687934 } \\
\text { Si,0.0001118097,-0.0008448436,2.6773058439 } \\
\text { Si,1.8061360651,0.0003690649,1.129585403 } \\
\text { Si,1.8061416368,0.0004471041,-1.129768811 } \\
\text { Si,-0.0001119121,-0.000937906,-2.6773058021 }\end{array}$ \\
\hline & MP2/6-311++G(d,p) & -1733.4814438 & $\begin{array}{l}\text { Si,-1.7372579712,0.0000393252,-1.1325221768 } \\
\text { Si,-1.7371854229,-0.0000224811,1.132815591 } \\
\text { Si,0.000192767,-0.000044829,2.6964543129 } \\
\text { Si,1.7372581327,-0.0000540976,1.1325224345 } \\
\text { Si,1.7371858989,0.0000075379,-1.1328154353 } \\
\text { Si,-0.0001934045,0.0000745446,-2.6964547263 }\end{array}$ \\
\hline & MP2/6-311++G(3df,3pd) & -1733.6754131 & $\begin{array}{l}\text { Si,-1.696016345,-0.000014333,-1.1341846693 } \\
\text { Si,-1.6959993448,-0.0000066272,1.1343541338 } \\
\text { Si,0.0001068144,0.0001461217,2.7419073036 } \\
\text { Si,1.6960156637,-0.0000773358,1.1341847097 } \\
\text { Si,1.6960000065,-0.0000848915,-1.1343541113 } \\
\text { Si,-0.0001067947,0.0000370658,-2.7419073664 }\end{array}$ \\
\hline$c-\mathrm{Si}_{6}{ }^{6-}$ & B3LYP/6-311++G(d,p) & -1735.6262862 & $\begin{array}{l}\text { Si,-2.1083619263,0.,-1.2172633257 } \\
\text { Si,-2.1083619263,0.,1.2172633257 } \\
\text { Si,0.,0.,2.4345266515 } \\
\text { Si,2.1083619263,0.,1.2172633257 } \\
\text { Si,2.1083619263,0.,-1.2172633257 } \\
\text { Si,0.,0.,-2.4345266515 }\end{array}$ \\
\hline
\end{tabular}


MP2/6-311++G(d,p)

B3LYP/6-311++G(3df,3pd)

MP2/6-311++G(3df,3pd)
$-1732.589581$

$-1735.6505928$

$-1732.7932311$
Si,-2.0390484828,-0.2442546119,-1.1892718824 Si,-2.0411958029,-0.2274434102,1.1886251552 Si, $0.0279531136,-0.2444069835,2.3604028282$ $\mathrm{Si}, 2.0390560946,0.2442268624,1.1892691765$ Si,2.0411935478,0.2274342163,-1.1886283715 Si,-0.0279584704,0.2444439269,-2.3603969059

Si,-2.0847818278,0.,-1.2036493495 Si,-2.0847818278,0.,1.2036493495 Si, $0 ., 0 ., 2.407298699$

Si,2.0847818278,0.,1.2036493495 Si,2.0847818278,0.,-1.2036493495 Si,0.,0.,-2.407298699

Si,-2.0598136052,0.,-1.1892339395 Si,-2.0598136052,0.,1.1892339395 Si,0.,0.,2.3784678789

$\mathrm{Si}, 2.0598136052,0 ., 1.1892339395$ Si,2.0598136052,0.,-1.1892339395 Si, $0 ., 0 .,-2.3784678789$

\begin{tabular}{|c|c|c|c|c|c|c|}
\hline \multirow{20}{*}{$\mathrm{Si}_{4}(\mathrm{NH} 2)_{2}$} & \multirow[t]{10}{*}{ B3LYP/6-311++G(3df,3pd) } & \multirow[t]{10}{*}{-1269.9776819} & \multirow{2}{*}{\multicolumn{4}{|c|}{$\begin{array}{l}\text { Si,-0.9113288114,0.0002217012,-2.9571563729 } \\
\text { Si,-0.8953311713,0.0001772385,-0.5671035293 }\end{array}$}} \\
\hline & & & & & & \\
\hline & & & \multicolumn{4}{|c|}{$\mathrm{Si}, 0.895342436,0.0000801684,0.5671001324$} \\
\hline & & & \multicolumn{4}{|c|}{$\mathrm{Si}, 0.9113229711,-0.0000410705,2.9571529699$} \\
\hline & & & \multicolumn{4}{|c|}{$\mathrm{N},-2.6011356763,-0.0002478247,-3.2676519415$} \\
\hline & & & \multicolumn{4}{|c|}{$\mathrm{H},-2.9448260936,-0.000325277,-4.2171579967$} \\
\hline & & & \multicolumn{4}{|c|}{$H,-3.3560221558,-0.0004721479,-2.5985340533$} \\
\hline & & & \multicolumn{4}{|c|}{ N,2.6011274912,-0.0003747014,3.2676615561 } \\
\hline & & & \multicolumn{4}{|c|}{ H,2.9448103473,-0.0004829745,4.2171703541 } \\
\hline & & & \multicolumn{4}{|c|}{ Н,3.3560192566,-0.0004944428,2.598549592 } \\
\hline & MP2/6-311++G(3df,3pd) & -1267.7999295 & \multicolumn{4}{|c|}{ Si,-0.9003978997,-0.0682461065,-2.9516892444 } \\
\hline & & & \multicolumn{4}{|c|}{ Si,-0.8809469394,-0.1608044247,-0.5747603355 } \\
\hline & & & \multicolumn{4}{|c|}{$\mathrm{Si}, 0.8809473475,0.1608699353,0.5747581714$} \\
\hline & & & \multicolumn{4}{|c|}{$\mathrm{Si}, 0.9003881846,0.0682587311,2.951683599$} \\
\hline & & & \multicolumn{4}{|c|}{$\mathrm{N},-2.5550439079,-0.4023183302,-3.2440045609$} \\
\hline & & & \multicolumn{4}{|c|}{$\mathrm{H},-2.9059979573,-0.437191862,-4.1904736358$} \\
\hline & & & \multicolumn{4}{|c|}{ H,-3.2778399882,-0.5812962481,-2.563856244 } \\
\hline & & & \multicolumn{4}{|c|}{$\mathrm{N}, 2.5550569048,0.4022057053,3.2440154189$} \\
\hline & & & \multicolumn{4}{|c|}{ H,2.9060084272,0.43702988,4.1904872863 } \\
\hline & & & \multicolumn{4}{|c|}{ H,3.2778688379,0.5811527116,2.5638759218 } \\
\hline \multirow[t]{7}{*}{ BeSi $_{6}$} & MP2/6-311++G(d,p) & -1748.745055 & \multirow{7}{*}{\multicolumn{4}{|c|}{$\begin{array}{l}\mathrm{Si}, 0,0.0000000439,2.2495613162,-0.0067505121 \\
\mathrm{Si}, 0,1.9481772692,1.1247806201,-0.0067505121 \\
\mathrm{Si}, 0,1.9481772253,-1.1247806961,-0.0067505121 \\
\mathrm{Si}, 0,-0.0000000439,-2.2495613162,-0.0067505121 \\
\mathrm{Si}, 0,-1.9481772692,-1.1247806201,-0.0067505121 \\
\mathrm{Si}, 0,-1.9481772253,1.1247806961,-0.0067505121 \\
\mathrm{Be}, 0,0 ., 0 ., 0.1417607536\end{array}$}} \\
\hline & & & & & & \\
\hline & & & & & & \\
\hline & & & & & & \\
\hline & & & & & & \\
\hline & & & & & & \\
\hline & & & & & & \\
\hline \multirow{12}{*}{$\mathbf{P}_{6}{ }^{4+}$} & \multirow[t]{6}{*}{ MP2/6-311++G(d,p) } & -2042.7669154 & $P$ & -1.84176 & 0. & -1.06334 \\
\hline & & & $\mathrm{P}$ & -1.84176 & 0. & 1.06334 \\
\hline & & & $\mathrm{P}$ & 0. & 0. & 2.12668 \\
\hline & & & $\mathrm{P}$ & 1.84176 & 0. & 1.06334 \\
\hline & & & $\mathrm{P}$ & 0. & 0. & -2.12668 \\
\hline & & & $\mathrm{P}$ & 1.84176 & 0. & -1.06334 \\
\hline & MP2/6-311++G(3df,3pd) & -2042.997513 & $P$ & -1.82803 & 0. & -1.05541 \\
\hline & & & $\mathrm{P}$ & -1.82803 & 0. & 1.05541 \\
\hline & & & $P$ & 0. & 0. & 2.11083 \\
\hline & & & $P$ & 1.82803 & 0. & 1.05541 \\
\hline & & & $\mathrm{P}$ & 0. & 0. & -2.11083 \\
\hline & & & $\mathrm{P}$ & 1.82803 & 0. & -1.05541 \\
\hline
\end{tabular}

